\title{
Antropologia física na primeira metade do século XX e a proposta tipológica de Earnest A. Hooton
}

Physical anthropology in the first half of the 20th century and the typological proposal of Earnest A. Hooton

Resumo

Apesar de ser considerado um dos fundadores da antropologia física, Earnest A. Hooton é uma figura pouco referenciada no contexto sul-americano. Suas contribuições para a bioarqueologia e estudo da variabilidade biológica das populações não foram isentas de críticas. Considerado por alguns como um representante claro do racismo e da eugenia, e por outros como um acadêmico e mentor que treinou toda uma geração de antropólogos físicos, ele fez importantes contribuições para a antropologia do período entre-guerras. Portanto, é interessante discutir o perfil desse cientista no contexto de seu tempo. O objetivo deste artigo é revisar a proposta metodológica de Earnest A. Hooton para a análise e classificação de grupos humanos, como parte dos debates científicos que ocorreram na Antropologia Física do final do século XIX e início do século XX os quais tentavam, sem muito sucesso, superar o interesse pela classificação tipológica das populações que dominou nas décadas anteriores e que teve influencia na América do Sul durante a primeira metade do século XX, especialmente porque estavam em curso estudos somatológicos que buscavam explicar a grande heterogeneidade biológica e cultural.
Juliana Gómez-Mejía

PhD en Ciencias Biológicas.

Docente Departamento de

Antropología y Sociología,

Universidad de Caldas.

Manizales, Colombia.

\juliana.gomez@ucaldas.edu.co

(1) ORCID: 0000-0003-3620-1798

$\triangle 1$ Google Scholar

Palavras-chave: racismo, eugenia, antropologia física, variabilidade biológica humana, somatologia. 


\section{ABstRact}

Despite being considered one of the founders of physical anthropology, Earnest A. Hooton is a poorly referenced figure in the South American context. His contributions to bioarcheology and the study of the biological variability of populations have not been exempt of criticism. Considered by some to be a clear representative of racism and eugenics, and by others as an academic and mentor who trained a whole generation of physical anthropologists, he made important contributions to anthropology in the interwar period. Therefore, it is interesting to discuss the profile of this scientist in the context of his time. The purpose of this article is to review Earnest A. Hooton's methodological proposal for the analysis and classification of human groups as part of the scientific debates that took place in physical anthropology in the late 19th and the early 20th centuries and which attempted, without much success, to overcome the interest in the typological classification of human groups that dominated in previous decades and that had an influence in South America during the first half of the 20th century, especially since somatological studies were being carried out that sought to explain the great biological and cultural diversity.

Keywords: racism, eugenics, physical anthropology, human biological variability, somatology.

\section{REsUmen}

A pesar de ser considerado como uno de los fundadores de la antropología física, Earnest A. Hooton es una figura poco referenciada en el contexto suramericano. Sus aportes a la bioarqueología y al estudio de la variabilidad biológica de las poblaciones no han estado exentos de críticas. Considerado por algunos como un claro representante del racismo y de la eugenesia, y por otros como un académico y mentor que entrenó a toda una generación de antropólogos físicos, hizo aportes importantes a la antropología del período entre guerras. Por lo tanto, resulta interesante discutir el perfil de este científico en el contexto de su época. El objetivo del presente artículo es presentar la propuesta metodológica de Earnest A. Hooton para el análisis y clasificación de grupos humanos, como parte de los debates científicos que se daban en la antropología física de finales del siglo XIX y de inicios del siglo $\mathrm{XX}$, los cuales intentaban, sin mucho éxito, superar el interés de la clasificación tipológica de los grupos humanos que dominó durante las décadas previas y que tuvo influencia en Suramérica durante la primera mitad del siglo XX, especialmente porque estaban en curso estudios somatológicos que querían dar cuenta de la gran heterogeneidad biológica y cultural.

Palabras clave: racismo, eugenesia, antropología física, variabilidad biológica humana, somatología. 


\section{Earnest A. Hooton: pioneiro da antropologia física}

antropologia física ${ }^{1}$ tem sido amplamente dedicada à obser-
vação e medição do corpo humano, com o objetivo de regis-
trar diferenças visíveis e quantificáveis entre indivíduos e grupos humanos, com base na definição de pontos anatômicos, diâmetros, comprimentos, profundidades e índices que respondem pelas formas, estruturas, dimorfismo sexual e ontogenético e composição corporal dos indivíduos. A variabilidade morfológica e a frequência com que certas características aparecem nas populações tem sido utilizadas pelos antropólogos para caracterizar e diferenciar algumas populações de outras, geralmente aqueles caracteres que oferecem ampla variabilidade e que têm uma base genética forte.

Durante a primeira metade do século XX, a Antropologia Física norte-americana esteve dominada por três pessoas: Franz Boas (1858-1942), Aleš Hrdlička (1869-1943) e Earnest A. Hooton (1887-1954). Porém, esse último é menos conhecido no contexto da América do Sul, no qual Boas destaca-se pelos aportes na área sociocultural e linguística, e Hrdlička, nos estudos sobre povoamento americano (Little y Kennedy, 2010).

Hooton se formou inicialmente em literatura clássica no Lawrence College (B.A. 1907) e na Universidade de Wisconsin (M.A. 1908; Ph.D. 1911). Depois, obteve seu Bacharelado em Antropologia (1912) e Bachelor of Letters (1913) na Universidade de Oxford. Sua formação em letras e não em medicina, permitiu-lhe explorar áreas que atualmente seriam consideradas multidisciplinares. Começou trabalhar na Universidade de Harvard, em 1913, como professor de antropologia física e muitos cientistas concordam que sua principal qualidade era sua excelente habilidade no ensino e sua capacidade de transmitir a seus estudantes a paixão pela ciência e a inspiração para os assuntos de antropologia física (Giles, 2010; Shapiro, 1954). Essa habilidade como professor favoreceu que Hooton formasse, até sua morte, uma quantidade importante de alunos de doutorado. A maioria deles dominou a disciplina antropológica com importantes aportes até a década de 80 , sendo os representantes da biologia humana com perspectiva biocultural nos Estados Unidos e ocupando espaços acadêmicos de grande reconhecimento como no American Journal of Physical

\footnotetext{
Em contraste, o termo antropologia biológica surgiu a partir dos avanços em genética e biologia molecular da década de 1950 e refere-se ao estudo da biologia humana desde uma perspectiva evolutiva com ênfase na relação entre biologia e cultura. Leva em conta aspectos que permitem estudar problemas centrados na genética, biologia evolutiva, nutrição, adaptação fisiológica, crescimento e desenvolvimento (Jurmain, Kilgore, y Trevathan, 2011).
} 
Anthropology (AJPA) e na American Association of Physical Anthropology (AAPA) (Buikstra y Beck, 2006; Little y Kennedy, 2010).

O período entre 1918 e 1930 esteve marcado pelo aumento do racismo e o interesse na eugenia. O movimento eugênico ganhou força a partir de 1890 e procurava "melhorar" a espécie humana e preservar a pureza "racial" a partir da reprodução planejada. A maioria dos eugenistas apoiou leis anti-miscigenação que proibiam casamentos inter-raciais. Outros eram ainda mais radicais, aceitando medidas como a esterilização (American Anthropological Association, 2007). Atualmente, existe um forte debate acadêmico sobre se as ideias de Hooton eram ou não racistas e eugênicas (Giles, 2012), e seu papel na luta contra o racismo durante a Segunda Guerra Mundial (Giles, 2010), considerando que Hooton foi dos poucos antropólogos que apoiou Franz Boas desde cedo quando falou em contra do racismo nazista (Little, 2010).

Os interesses iniciais de Hooton se concentravam na arqueologia, mas depois ele voltou toda sua atenção para assuntos biológicos relacionados à antropologia. Os trabalhos The Ancient Inhabitants of the Canary Islands (1925) e The Indians of Pecos Pueblo. A Study of Their Skeletal Remains (1930), dão início aos estudos da saúde e de doenças em populações passadas sob uma perspectiva ecológica onde pela primeira vez foi utilizada uma abordagem epidemiológica inauguradora da paleopatologia moderna. Esta pesquisa utilizou de forma inovadora, estatística simples com múltiplos marcadores de saúde e promoveu a interdisciplinaridade nas interpretações dos restos ósseos (Armelagos y Van Gerven, 2003; Goodman, 1993).

A partir de 1930, Hooton passou de trabalhar quase exclusivamente com ossos humanos para observar a variação das populações vivas. Um dos textos mais populares foi Up from the ape (1931). Com um estilo próprio e pouco especializado o livro conseguia atingir um público mais abrangente. Esse estilo está presente também em múltiplos artigos e livros como: Apes, Men and Morons (1937), Twilight of Man (1939c), Why Men Behave Like Apes and Vice Versa (1940), Man's Poor Relations (1942), e Young Man, You Are Normal (1945). Outras linhas de pesquisa, mais controvérsias devido às tendências consideradas como racistas, incluíram estudos somatológicos ${ }^{2}$ e sobre as características físicas dos criminosos

\footnotetext{
A somatología estudava a forma do corpo humano e sua relação com aspectos do comportamento, entre eles, a capacidade de cometer algum tipo de crime. Os conceitos de morfotipo, psicótipo, biótipo, somatótipo e constituição são levados em consideração, para fazer uma descrição e representação somatotipológica, na qual a constituição é a soma total dos caracteres morfológicos, psicológicos e fisiológicos de um indivíduo. A técnica de Sheldon (1940) iniciou o desenvolvimento da escola norte-americana somatotipologica.
} 
da América do Norte com trabalhos como Crime and the Man (1939a) e The American Criminal (1939b). Nesses trabalhos, também se destacam os desenhos que representavam a relação entre "raça" e comportamentos criminosos (Figura 1).
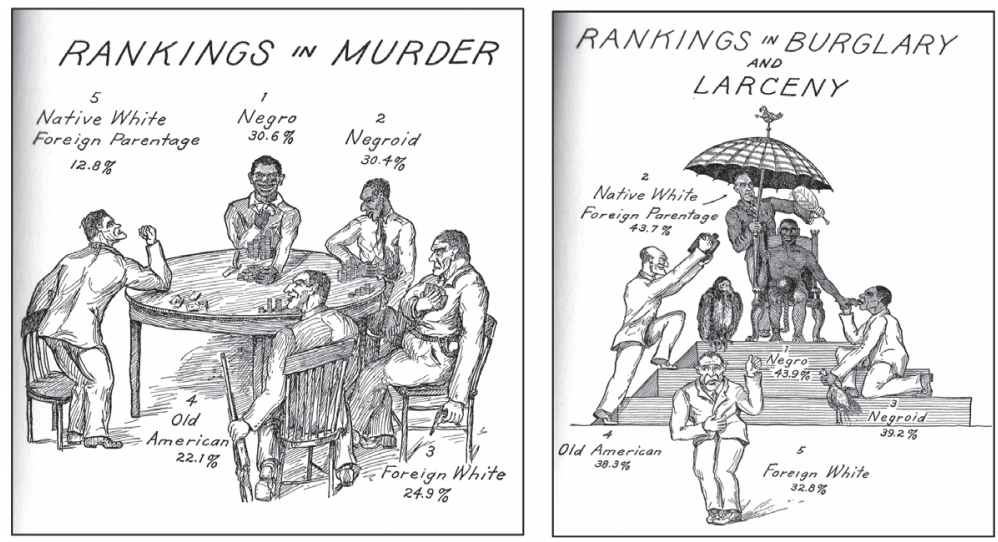

Figura 1. Rankings em assassinato, roubo e furto segundo a raça.

Fonte: Hooton, 1939a.

\section{A proposta de análise e classificação racial de Earnest A. Hooton}

Desde os séculos XVIII e XIX, o interesse estava na classificação tipológica dos grupos humanos, também conhecida como craniologia, e não na variabilidade ${ }^{3}$. Continuava persistindo a ideia de escalas inferiores e superiores, assim como categorias fixas que ignoravam as variações. A tipologia, como método, era uma forma de reduzir o espectro de variação a um conjunto de categorias que fossem de fácil manejo. Porem, desde uma perspectiva filosófica, era um olhar de estereótipos com dois problemas básicos: 1. Os tipos eram idealizados, portanto ninguém do mundo real conseguia encaixar, e 2. Aplicar um "tipo" para descrever uma população, não permite enxergar a variação que existe. Portanto, o conjunto de "tipos" termina parecendo algo real e a variabilidade uma abstração (Johnston y Little, 2012).

O artigo "Methods of racial analysis" (1926) publicado na revista Science, procurava ser um aporte aos debates científicos do final do século XIX e de inícios do século XX apresentando uma discussão do conceito biológico e antropológico de "raça". Para Hooton (1926):

O anatomista alemão Johann Friedrich Blumenbach (1752-1840) já tinha sugerido noção sobre a natureza continua da variação humana y definiu a ideia de "raça" em De generis humani varietate nativa (1775) donde estabelecia que as classificações raciais fossem arbitrarias, embora ele classificasse os humanos em cinco raças. 
Uma raça é uma grande divisão da humanidade, seus membros, embora variados individualmente, são caracterizados como um grupo pela combinação de determinados atributos morfológicos e métricos, principalmente não-adaptativos, que foram derivados a partir de sua descendência comum. (p. 76)

Os seguintes caracteres corporais eram considerados por Hooton como as principais variações não adaptativas: a forma, a cor e quantidade de cabelo e sua distribuição em áreas, a cor dos olhos e a forma das dobras da pálpebra, a forma das cartilagens nasais, a forma dos lábios e da orelha, a prominência do queixo, a largura da cabeça em relação a seu comprimento, o comprimento do rosto, os padrões suturais, a presença ou ausência de um tubérculo pós-glenóide e uma fossa ou tubérculo na faringe, prognatismo, a forma do dente incisivo, a forma da borda vertebral da escápula, a presença ou ausência do processo supracondilar ou forâmen no úmero, a largura do antebraço em relação ao braço, o grau de arqueamento do rádio e ulna e a largura da perna em relação à coxa. Outras características que podiam ser usadas como critério racial por serem persistentes em várias linhagens eram: pigmentação da pele, largura do rosto, altura e largura do nariz, tamanho e proeminência dos zigomáticos, altura da cabeça, volume do cérebro, proporção do tórax, comprimento relativo das extremidades inferiores, comprimento relativo e ângulo de inclinação do osso calcanhar e tamanho e desenvolvimento dos músculos da panturrilha. Outras características facilmente modificáveis no individuo e no grupo através da ação de fatores ambientais e especialmente pela qualidade da nutrição, dieta, modo de andar e atividade física não seriam confiáveis, segundo Hooton, como critério de "raça".

No artigo, Hooton sugere a existência de "raças" primárias e secundárias. Uma "raça" primária era aquela que teria sido modificada unicamente pela operação de fatores evolutivos, incluindo a seleção de suas próprias variações intrínsecas e das modificações, adaptativas ou não, possivelmente causadas pelo estímulo ambiental. Uma "raça" secundária, ou composta, era aquela na qual a combinação característica e estabilizada de atributos morfológicos e métricos, tem sido resultado de uma mistura longa e continua entre duas ou mais raças primárias, em uma determinada área relativamente isolada. Por tanto, a maior parte da população humana estaria conformada por "raças" secundárias e as primárias, representadas unicamente por grupos consanguíneos no interior de áreas com pouco contato racial. A relação que apontava entre "raças" e comportamento fica clara quando fala: "Se as raças diferem quantitativamente em inteligência e qualitativamente em características psicológicas, essas diferenças podem ser estabelecidas unicamente pela 
segregação dos tipos raciais puros e pela subsequente pesquisa de seus atributos psicológicos" (p. 81).

Contudo, várias das posições do Hooton têm sido classificadas por diversas fontes como racistas e eugênicas (American Anthropological Association, 2007; Giles, 2012). No entanto, fica claro o interesse do Hooton em fazer esclarecimentos sobre o assunto racial, considerando as confusões de conceitos existentes na época e sua premissa de que todas as pessoas do seu tempo eram racialmente misturadas. Para Hooton (1926) o termo "raça", aplicado ao homem, era comumente utilizado sem um significado acurado ou mesmo nem bem definido implicando em uma confusão de critérios:

Falar da raça "branca" é assumir que raça é uma questão de pigmentação da pele; referir-se à "raça judia" é diferenciar raça com base na religião; a "raça latina" implica um critério linguístico, e finalmente qualquer referência a uma "raça irlandesa" deve significar uma raça caracterizada tanto pela posição geográfica, ou na sua falta, pelo temperamento. (p. 75)

Mais à frente, esclarece que: "Certamente, se a fertilidade nos entrecruzamentos é considerada como a prova das espécies, todas as variedades atuais de homem devem ser designadas em um único grupo" (p. 76).

Porém, o propósito do artigo do Hooton era apresentar uma metodologia para fazer diagnósticos "raciais", e nessa linha o trabalho continuava ligado aos estudos tipológicos e classificatórios que predominavam desde o século anterior. O método de analise racial proposto por Hooton, destacava as vantagens e desvantagens de utilizar pessoas vivas em comparação com o estudo de esqueletos, entre elas, a possibilidade de incluir caraterísticas significativas que estão ausentes no material esquelético, como a forma das partes moles dos olhos, nariz e boca, a forma e distribuição do cabelo e a pigmentação. Por outro lado, algumas desvantagens em comparação aos esqueletos, consistiam em:

As medidas das partes moles são menos acuradas do que as medições no esqueleto e as características ósseas mais significativas não podem ser observadas ou medidas in vivo. Alguns caracteres notáveis e óbvios dos indivíduos vivos podem enganar ao investigador em sua análise racial. A forma e a cor do cabelo são, com frequência, alteradas radicalmente por meios artificiais. O fenômeno de dominância tende a ocultar relações em raças compostas, especialmente na pigmentação e na forma do cabelo e partes moles. 
O cabelo tingido com pigmento amarelo-marrom e a dominância do cabelo liso, grosso e preto, têm, por exemplo, na minha opinião, criado uma impressão equivocada da homogeneidade física do Índio Americano. As vantagens e desvantagens de analisar elementos raciais em material esquelético são naturalmente opostas àquelas que se obtém em casos de pessoas vivas. Nosso atual conhecimento imperfeito da correlação das variações nas partes moles com aquelas do esqueleto torna incertas muitas das determinações raciais em ambos os casos. Porém, na investigação de tipos de homens antigos e extintos e de populações do passado, dependemos naturalmente do material ósseo. Minha opinião é que as características raciais estão mais bem definidas no esqueleto do que nas partes moles. Esse parece ser o caso particularmente do crânio. (p. 78)

Mesmo assim, a proposta metodológica apresentada no artigo, foi um claro antecedente para estudos posteriores de variabilidade biológica humana, mesmo que não fosse para classificar tipos de "raças". O chamado a levar em conta analises estatísticos e critérios como: presença ou ausência, grau de desenvolvimento e forma, são ainda utilizados nas observações morfológicas e nas analises bioantropológicas regulares. Aliás, Hooton parecia entender muito bem as limitações dos estudos tipológicos. Para ele, "combinações de médias grupais podem não representar nenhum tipo físico individual, mas apenas médias de caracteres variáveis independentes" (p. 79), portanto, a análise devia ser de grupos e não de indivíduos separados.

O artigo também considera de maneira importante o estudo de afinidade biológica entre grupos através de material esquelético, especialmente crânios. Segundo Hooton, em cada esqueleto poderiam ser feitas as medidas habituais e calculados os índices convencionais. As observações morfológicas relacionadas com caracteres não medíveis deviam ser cuidadosamente classificadas por graus e registradas em formatos apropriados. Depois de fazer as medições e observações, cada crânio era classificado em um tipo distinto, segundo a avaliação anatômica do observador. O seguinte passo era a redução estatística das medidas e índices para o grupo como um todo, separando os dois sexos. Esse procedimento incluía o calculo das médias, dos desvios padrões, dos coeficientes de variação e seus erros prováveis. Também eram calculadas as distribuições porcentuais das subdivisões dos índices. As observações morfológicas por graus também eram analisadas para o grupo inteiro e as porcentagens de cada grau para cada caractere era tabulado segundo o sexo. 
O passo seguinte era a comparação do grupo com outros grupos que tivessem dados comparativos disponíveis e que estiveram possivelmente relacionados, além de ter uma composição "racial" conhecida de forma mais ou menos acurada. A comparação era feita entre as diferenças das médias dos dois grupos para cada medida e índice. Se as diferenças entre as médias dos grupos com relação à determinada medida ou índice era menor que três vezes o erro provável, então os dois grupos não diferiam significativamente com relação à média dessa medida ou índice. Coincidências nas médias de duas ou três medidas ou índices não eram indicativas de relação entre os grupos. Porém, se um grande número de medidas e índices nos dois grupos apresentava diferenças mínimas, incrementa-se a probabilidade de que as duas populações tenham uma composição "racial" semelhante. Comparando vários grupos com aquele que está sob investigação, poderíamos, segundo Hooton, descobrir com qual desses o último grupo tem afinidade mais estreita. A proposta de Hooton encaixava parcialmente na escola dos biometricistas, que dedicaram-se principalmente às análises estatísticas das medidas antropométricas e à determinação das relações de tais medidas entre si e com várias condições ambientais. Sob a liderança de Karl Pearson, esses biometricistas desenvolveram ferramentas estatísticas precisas, pelas quais os erros incidentes ao processo de amostragem eram considerados, e as variabilidades e os coeficientes da relação das variáveis entre si eram calculados.

Finalmente, Hooton era precavido em métodos intuitivos e considerava os dados morfológicos e métricos obtidos adequadamente como mais confiáveis: "Se os antropólogos físicos querem obter resultados cientificamente válidos não devem conformar-se em apresentar tabelas de medições complementadas com suas reações pessoais pela via da interpretação" (p. 81).

A proposta metodológica de Hooton, estava no meio do debate em relação às outras implicações de uma classificação da humanidade orientada pelos atributos corporais (Little \& Kennedy, 2010). Havia também antropólogos que negavam a existência de correlatos culturais ou psicológicos de "raça", e muitas das variações somatológicas eram consideradas como efeitos do ambiente. A pigmentação era tratada por eles como resultado do clima, a estatura como uma consequência da nutrição e a forma da cabeça como uma manifestação da variação individual ou um subproduto de fatores de tamanho herdados separadamente.

Essa ideia de "raças" e tipos perdurou por algumas décadas a mais, até que começaram a ser incluídas ideias darwinianas e conceitos como 
variação populacional, adaptação, seleção e evolução (Johnston \& Little, 2012). Franz Boas, fez aportes destacados para a biologia humana, a partir do trabalho sobre migrantes da Europa nos Estados Unidos (Boas, 1912) e sobre a variação no crescimento e desenvolvimento de adolescentes (Boas, 1930). Ele demostrou que o ambiente era uma força importante e que tinha efeitos no tamanho e constituição, sendo a plasticidade a principal causa de variação humana (Little, 2010).

Mesmo sendo pouco estudado nos currículos de Antropologia, os estudos somatológicos e os critérios de classificação, tiveram impacto na América do Sul durante a primeira década do século XX, especialmente porque estavam em curso os debates sobre a formação das nacionalidades no meio de uma grande heterogeneidade biológica e cultural, produto de mestiçagem e migrações (Ribeiro, 1995). Por exemplo, no Brasil foram bastante populares durante este período "as investigações antropofísicas sobre grupos indígenas, negros, sertanejos e imigrantes europeus; os debates sobre miscigenação racial, imigração e eugenia; e os estudos de biotipologia, medicina legal e antropologia jurídica" (Souza \& Santos, 2012, p. 640). A ênfase nas características anatômicas, fisiológicas e psicológicas dos diferentes 'tipos raciais' brasileiros levaram a fortalecer toda uma escola de antropologia física no Brasil com representantes como: Edgard Roquette-Pinto, José Bastos de Ávila, Álvaro Fróes da Fonseca, Waldemar Berardinelli e Isaac Brown (entre outros), permitindo que fosse constituída uma biotipologia brasileira que procurava "medir e classificar a população de diferentes maneiras, determinando o temperamento, o 'tipo' corporal ou o 'normotipo do homem médio brasileiro" (Souza \& Santos, 2012, p. 641).

Na Colômbia, o interesse pela diversidade física de populações indígenas ganhou força a partir da década de 1940 quando pesquisadores como Milciades Chaves, Graciliano Arcila, Henry Lehmann, Paulette Marquer, Reichel Dolmatoff (entre outros) publicaram trabalhos sobre as características somatologicas dos grupos: Katio, Noanamá, Juan José, Chimilas, Guambiano-Kokonuco, Kwaiker, Guahibos e Pijao. Por outro lado, em países como na Argentina também foram comuns os enfoques biotipológicos durante a primeira metade de século XX, especialmente para "controlar" com mecanismos eugênicos os processos migratórios associados às grandes guerras (Vallejo \& Miranda, 2004). Da mesma forma, no Chile a influencia de pesquisadores como Waldemar Coutts durante as década de 1920 e 1930 fortaleceu os movimentos eugênicos (Sánchez, 2018). 
Ao longo deste artigo foi apresentado o perfil de Earnest A. Hooton e suas contribuições, com ênfase na proposta metodológica para a análise e classificação de grupos humanos. Com tendências racistas e eugênicas, próprias da antropologia da primeira metade do século XX, Hooton treinou toda uma geração de antropólogos físicos que se manteve ativa nos debates que tentavam superar a ênfase na classificação biotipológica. Mesmo assim, a influencia destas pesquisas na América do Sul ainda está por ser avaliada com maior detalhe, considerando a heterogeneidade biológica e cultural no contexto de formação das nacionalidades.

\section{Referências}

American Anthropological Association. (2007). Race: Are We So Different? Retrieved from http://www.understandingrace.org

Armelagos, G. y Van Gerven, D. (2003). A Century of Skeletal Biology and Paleopathology: Contrasts, Contradictions, and Conflicts. American Anthropologist, 105(1), 53-64.

Boas, F. (1912). Changes in the bodily form of descendants of immigrants. American Anthropologist, 14(3), 530-562.

Boas, F. (1930). Observations on the growth of children. Science, 72(1854), 44-48.

Buikstra, J. y Beck, L. (2006). Bioarchaeology The Contextual Analysis of Human Remains. Elsevier Inc.

Giles, E. (2010). Principal Figures in Physical Anthropology Before and During World War II. En M. Little y K. Kennedy (eds.), Histories of American Physical Anthropology in the Twentieth Century (pp. 141-154). United Kingdom: Lexington Books.

Giles, E. (2012). Two faces of earnest A. Hooton. American Journal of Physical Anthropology, 149, 105-113. https://doi.org/10.1002/ajpa.22162

Goodman, A. (1993). On the Interpretation of Health From Skeletal Remains. Current Anthropology, 34(3), 281-288.

Hooton, E.A. (1925). The ancient inhabitants of the Canary Islands (Vol. 7). Corinthian Press.

Hooton, E.A. (1926). Methods of racial analysis. Science, LXIII(1621), 75-81.

Hooton, E.A. (1930). The Indians of Pecos Pueblo: a study of their skeletal remains. Department of Archaeology, Phillips Academy, Andover, Mass.

Hooton, E.A. (1931). Up from the Ape. New York: Macmillan.

Hooton, E.A. (1937). Apes, Men and Morons. New York: G. P. Putnam's sons.

Hooton, E.A. (1939a). Crime and the Man. Cambridge, Massachusetts: Harvard University Press.

Hooton, E.A. (1939b). The American criminal: an anthropological study. Cambridge, Massachusetts: Harvard University Press.

Hooton, E.A. (1939c). Twilight of Man. New York: G. P. Putnam's sons.

Hooton, E.A. (1940). Why Men Behave Like Apes and Vice Versa. Princeton: Princeton University Press. 
Hooton, E.A. (1942). Man's Poor Relations. New York: Doubleday.

Hooton, E.A. (1945). Young Man, You Are Normal. New York: G. P. Putnam's sons.

Johnston, F. y Little, M. (2012). History of Human Biology in the United States of America. En S. Stinson, B. Bogin, \& D. O’Rourke (eds.), Human Biology: An Evolutionary and Biocultural Perspective (Second Edi, pp. 23-50). New Jersey: John Wiley \& Sons, Inc.

Jurmain, R, Kilgore, L. y Trevathan, W. (2011). Essentials of Physical Anthropology. 8 [sup] th ed. USA: Wadsworth, Cengage Learning, 8-20.

Jurmain, R., Kilgore, L. y Trevathan, W. (2009). Essentials of Physical Anthropology. Wadsworth Cenage Learning.

Little, M. (2010). Franz Boas's Place in American Physical Anthropology and Its Institutions. En M. Little \& K.A.R. Kennedy (eds.), Histories of American Physical Anthropology in the Twentieth Century (pp. 55-85). United Kingdom: Lexington Books.

Little, M. y Kennedy, K. (2010). Histories of American Physical Anthropology in the Twentieth Century. United Kingdom: Lexington Books.

Ribeiro, D. (1995). O povo brasileiro: a formação eo sentido do Brasil. São Paulo: Companhia das Letras.

Sánchez, M. (2018). Sexo, eugenesia y política: Waldemar Coutts (Chile, 1895-1959). Revista de Historia, 25(1), 109-130.

Shapiro, H.L. (1954). Earnest Albert Hooton 1887-1954. American Anthropologist, 56(6), 1081-1084. https://doi.org/10.1525/aa.1954.56.6.02a00090

Sheldon, W.H., Stevens, S.S. y Tucker, W.B. (1940). The varieties of human physique. New York: Harper.

Souza, V.S. de, y Santos, R.V. (2012). Corpos, medidas e nação: antropologia física na capital da República brasileira na primeira metade do século XX. Boletim Do Museu Paraense Emílio Goeldi. Ciências Humanas, 7(3), 639-643.

Vallejo, G., y Miranda, M. (2004). Los sabores del poder: eugenesia y biotipología en la Argentina del siglo XX. Revista de Indias, 64(231), 425-444. 